\title{
ARTICLE AZD9291 inactivates the PRC2 complex to mediate tumor growth inhibition
}

\author{
Kai-li Zhang ${ }^{1,2}$, Qian-qian Shen ${ }^{1}$, Yan-fen Fang ${ }^{1}$, Yi-ming Sun ${ }^{1}$, Jian Ding ${ }^{1}$ and Yi Chen ${ }^{1}$
}

Deregulated Polycomb repressive complex 2 (PRC2) is intimately involved in tumorigenesis and progression, making it an invaluable target for epigenetic cancer therapy. Disrupting the EZH2-EED interaction, which is required for PRC2 enzymatic activity, is a promising strategy for cancer treatment. However, this kind of inhibitors are still limited. The in-cell protein-protein interaction screening was conducted for approximately 1300 compounds by NanoBRET technology. Co-immunoprecipitation (Co-IP), protein thermal shift assay (PTSA), and cellular thermal shift assay (CETSA) were performed to investigate the regulation of PRC2 by AZD9291. The anti-tumor effects of AZD9291 on breast cancer (BC) cells and diffuse large B-cell lymphoma (DLBCL) cells were detected. MicroRNA array assay, luciferase reporter assay, and qRT-PCR were conducted to identify the interaction and regulation among AZD9291, EZH2, and miR-34a. We discovered that, AZD9291, a potent and selective EGFR inhibitor, disrupted the interaction of EZH2-EED, leading to impairment of PRC2 activity and downregulation of EZH2 protein. In addition, AZD9291 declined EZH2 mRNA expression via upregulating the expression of a tumor suppressor, miR-34a. Our results suggest that AZD9291 can serve as a lead compound for further development of antagonist of PRC2 protein-protein interactions and EZH2 mRNA may be a direct target of miR-34a through non-canonical base pairing.

Keywords: PRC2; EZH2-EED; AZD9291; miR-34a; non-canonical base pairing

Acta Pharmacologica Sinica (2019) 40:1587-1595; https://doi.org/10.1038/s41401-019-0248-2

\section{INTRODUCTION}

Polycomb repressive complex 2 (PRC2) and its histone H3 lysine 27 methylation activity are crucial for repressing target genes expression, which play a critical role in cancer initiation, progression, and metastasis. Previous studies showed that PRC2 was frequently dysregulated in multiple human cancers [1-4]. The enzymatic unit of PRC2 resides in the enhancer of zeste homolog 2 (EZH2) subunit's SET region, which mediates silencing of target genes via local chromatin reorganization. Furthermore, high levels of H3K27me3 have been found in both hematological malignancies and solid cancers, due to EZH2 overexpression and/or EZH2 mutation [5-7]. These evidence highlight the development of PRC2 inhibitors as a therapeutic approach for cancer treatment. Indeed, several S-adenosyl- $L$-methionine (SAM)-competitive compounds are currently in clinical trials, and have demonstrated efficacy in treating hematological malignancy.

In addition, PRC2 is an obligate multimeric complex, and indeed its components all engage the chromatin substrate in the course of $\mathrm{H} 3 \mathrm{~K} 27$ me3 deposition by EZH2 [8]. EZH2 adopts an autoinhibited conformation when on its own, and its catalytic activity and in vivo stability both require binding to two other core PRC2 subunits, EED and SUZ12 [8]. H3K27me3 recognition by EED is essential in stimulating basal PRC2 activity and propagating H3K27 methylation in repressive chromatin for gene silencing. And the binding of $\mathrm{H} 3 \mathrm{~K} 27 \mathrm{me} 3$ to EED causes a conformational change of the stimulation-responsive motif in $\mathrm{EZH} 2$, leading to the enhanced catalytic efficiency, which was illustrated by crystal structures of the PRC2 complex. This implicated finding PRC2 inhibitors disabled the PRC2 complexes in cancer by destroying protein interactions is an alternative strategy. A stabilized a-helix of EZH2 (SAM-EZH2) peptide has been reported that it selectively inhibits H3 Lys27 tri-methylation in PRC2-dependent MLL-AF9 leukemia cells by disrupting the EZH2-EED complex [9]. Moreover, EED226 and A-395 represent small molecular antagonists of PRC2 protein-protein interactions for disruption of the PRC2 complex $[10,11]$.

In the present study, we aimed at finding a pharmacologic approach to suppression of PRC2 function by targeted disruption of PRC2 complex. We first found that AZD9291, a covalent epidermal growth factor receptor (EGFR) tyrosine kinase inhibitor, which has been approved by the US FDA for the treatment of nonsmall-cell lung cancer (NSCLC) patients with EGFR T790M [12], disrupted the interaction between EZH2 and EED, impaired the PRC2 complex and inhibited cancer cells proliferation and mobility. Then, we explored the potential regulatory mechanisms and found that AZD9291 also regulated the expression of EZH2 via upregulating miR-34a. Additionally, miR-34a may directly target the mRNA of EZH2 through a non-canonical binding. Therefore, AZD9219 may provide a therapeutic option and indicate us to further improve the efficacy of PRC2 inhibitors.

\footnotetext{
${ }^{1}$ Division of Anti-Tumor Pharmacology, State Key Laboratory of Drug Research, Shanghai Institute of Materia Medica, Chinese Academy of Sciences, Shanghai 201203, China and ${ }^{2}$ University of Chinese Academy of Sciences, Beijing 100049, China

Correspondence: Jian Ding (jding@simm.ac.cn) or Yi Chen (ychen@simm.ac.cn)
}

Received: 6 March 2019 Accepted: 5 May 2019

Published online: 6 June 2019 


\section{MATERIALS AND METHODS}

Cell lines and culture

The cell lines BT-549 and Daudi were purchased from the cell bank of the Chinese Academy of Sciences (Shanghai, China). The cell lines BT20, T47D, Hs578t, HCC38, HCC1143, HCC1428, HCC1395, HCC1954, HBL-100, MDA-MB-175, MDA-MB-453, MDA-MB-231, MDA-MB-468, MCF-7, S1, ZR-75-1, ZR-75-30, CCRF-CEM, HH, HEK293, HEK293T, Pfeiffer, Raji, Ramous, and Mino were purchased from the American Type Culture Collection (ATCC, Manassas, VA, USA). CAL-51, EFM-19, HT, DB, KARPAS-422, L-428, RL, SU-DHL-4, SU-DHL-5, SU-DHL-8, U2932, WSU-DL-CL2, WILL-1, and WILL-2 were obtained from Deutsch Sammlung von Mikroorganismen und Zellkulturen (DSMZ, Braunschweig, Germany). BT549, HCC1937, and HCC1954 were cultured in RPMI-1640 medium (Gibco, Grand Island, NY, USA) with $2.5 \mathrm{~g} / \mathrm{L}$ glucose and $0.11 \mathrm{~g} / \mathrm{L}$ sodium pyruvate. BT20, CAL-51, HCC38, HBL-100, MCF-7, and S1 were cultured in low-glucose Dulbecco's modified Eagle's medium (Gibco). T47D, HCC1428, ZR-75-1, ZR-75-30, EFM-19, Ramos, L-428, and $\mathrm{U} 2932$ were cultured in RPMI-1640 medium. Hs578t, MDAMB-175 and MDA-MB-453, HEK293 and HEK293T were cultured in DMEM/F12 (Gibco). MDA-MB-231 and MDA-MB-468 were cultured in L-15 medium (Gibco). All above medium supplemented with $10 \%-15 \%$ FBS (Gibco) and $100 \mathrm{U} / \mathrm{mL}$ penicillin and $100 \mu \mathrm{g} / \mathrm{mL}$ streptomycin. CCRF-CEM, Daudi, HH, Raji, Mino, and Pfeiffer cells were cultured in RPMI-1640 medium supplemented with $10 \%-15 \%$ FBS and with $2.5 \mathrm{~g} / \mathrm{L}$ glucose and $0.11 \mathrm{~g} / \mathrm{L}$ sodium pyruvate. DB, RL, SU-DHL-4, SU-DHL-5, SU-DHL-8, KARPAS-422, WILL-1, and WILL-2 were cultured in RPMI-1640 medium with $20 \%$ FBS. MDA-MB-231 and MDA-MB-468 were cultured in air humidified incubator at $37^{\circ} \mathrm{C}$ and other cells were cultured in a $5 \% \mathrm{CO}_{2}$ humidified incubator at $37^{\circ} \mathrm{C}$. All cell lines were authenticated through analysis of short tandem repeats (Genesky Biotechnologies, Shanghai, China) and exactly matched the cell lines in the DSMZ and ATCC data bank.

Compounds, antibodies, and reagents

AZD9291 (HY-15772) and EPZ6438 (HY-13803) were purchased from the Med Chem Express (Monmouth Junction, NJ, USA); Astemizole (ab144583, Abcam, Cambridge, UK); MG-132 (S2619, Selleckchem, Houston, TX, USA), gefitinib (S1025, Selleckchem), afatinib (S1011, Selleckchem), EED226 (S8496, Selleckchem), both of them claimed to have more than $98 \%$ purity of the compound based on a highperformance liquid chromatography analysis. Human EGF factor (AF100-15) was purchased from PeproTech (Rocky Hill, NJ, USA).

Antibodies were used as follows: EZH2 (5246S, Cell Signaling Technology, CST, Danvers, MA, USA); EED (075530, Millipore, Bedford, MA, USA); SUZ12 (20366-1-AP, Proteintech, Rosemont, IL, USA); Beta Actin (HRP-60008, Proteintech); H3K27me3 (9733, CST); histone-H3 (17168-1-AP, Proteintech); H3K27me2 (9728, CST); H3K79me3 (4260, CST); H3K36me3 (4909, CST); H3K4me3 (9751, CST); ubiquitin (3933, CST).

Transfections with miR-34a mimics, miR-NC or inhibitor (GenePharma, Shanghai, China), siRNAs (GenePharma) and plasmids were performed using Lipofectamine RNAiMAX (Invitrogen, Carlsbad, CA, USA) and Lipofectamine 2000 (Invitrogen) respectively, according to the manufacturer's protocol. The sequences can be found in Table S2.

Bioluminescence resonance energy transfer (BRET) assay

Both EZH2 (NP_004447.2) and EED (NP_001294936.1) were inserted into the respective CMV-neo Flexi vectors, according to the manufacturer's instructions (NanoBRET PPI Flexi Starter System, Promega, Madison, WI, USA). Eight potential clones and eight potential donor/acceptor combinations were screened, and the best combination that pFN31K-EZH2 Nluc+pFC14K-EED HaloTag (EZH2 protein tagged with NanoLuc at N-terminal and EED protein tagged with HaloTag at C-terminal) was chosen to carry out the NanoBRET assay.
We carried out NanoBRET assays using a NanoBRET Nano-Glo detection System (N1662, Promega) according to the manufacturer's instructions. Briefly, HEK293T cells were co-transfected with HaloTag and NanoLuc fusions using Lipofectamine 2000 for approximately $20 \mathrm{~h}$ at $37^{\circ} \mathrm{C}, 5 \% \mathrm{CO}_{2}$. The transfected cells were trypsinized, washed with PBS, resuspended in assay medium (Opti-MEM I Reduced Serum Medium, no phenol red (Thermo Scientific, Waltham, MA, USA) $+4 \%$ FBS), re-plated to a 96-well plate, and incubated with HaloTag NanoBRET 618 Ligand (100 nM final concentration), and compounds overnight (18-24 h). Vehicle (DMSO) was added as negative control. NanoBRET Nano-Glo Substrate was added, then donor emission (460 $\mathrm{nm}$ ) and acceptor emission $(618 \mathrm{~nm})$ were measured using the SYNERGY 2 microplate reader (BioTek, Winooski, VT, USA). Raw NanoBRET ratio values with milliBRET units $(\mathrm{mBU})$ were calculated as RawBRET $=$ $618 \mathrm{~nm} \mathrm{Em/460} \mathrm{nm} \mathrm{Em} \times 1000$. Corrected NanoBRET ratio values were calculated as corrected BRET = RawBRET of experimental sample - RawBRET of no-ligand control sample.

\section{Western blot analysis}

Protein samples from cells were analyzed by SDS-PAGE and transferred to a nitrocellulose membrane (Bio-Rad, Hercules, CA, USA). The membrane was blocked with $5 \%$ milk for $1 \mathrm{~h}$ at room temperature, followed by incubation with the indicated primary antibodies at $4{ }^{\circ} \mathrm{C}$ overnight. The membrane was subsequently incubated with horseradish peroxidase (HRP)-conjugated goat anti-Mouse or anti-rabbit IgG (Jackson ImmunoResearch, West Grove, PA, USA) for $1 \mathrm{~h}$. Proteins were detected using Image Quant LAS4000 (GE Healthcare, Chicago, IL, USA).

Co-immunoprecipitation (Co-IP)

The experiments were carried out with lysates prepared with NP40 Lysis Buffer from HEK293T cells treated with AZD9291 or EED226 for $48 \mathrm{~h}$. The cell lysates were incubated with indicated antibody overnight at $4{ }^{\circ} \mathrm{C}$ and then Pierce Protein A/G Magnetic Beads (Thermo Scientific) were added. After $4 \mathrm{~h}$ incubation, the binding proteins were eluted from the beads with NP-40 Lysis Buffer and followed by Western blot analysis.

Protein thermal shift assay (PTSA)

The thermal shift assay was performed on a 7500 fast real-time PCR system (Applied Biosystems, Foster City, CA, USA) according to manufacturers' protocol. Each reaction solution containing $2 \mu \mathrm{M}$ EED, $5 \times$ SYPRO orange (Invitrogen), and testing compounds in $20 \mu \mathrm{L}$ of thermal shift buffer $(25 \mathrm{mM}$ PIPES, pH 6.2, $150 \mathrm{mM}$ $\mathrm{NaCl}, 1 \mathrm{mM}$ DTT) were heated from 25 to $95^{\circ} \mathrm{C}$ at $1 \%$ ramp rate. The change in the fluorescence intensities of SYPRO orange was monitored as a function of the temperature. Each reaction was repeated three times.

Whole cell cellular thermal shift assay (CETSA)

The thermal shift assay was performed on a 7500 fast real-time PCR system (Applied Biosystems, Foster City, CA, USA) according to manufacturers' protocol. Each reaction solution containing 2 $\mu \mathrm{M}$ EED, $5 \times$ SYPRO orange (Invitrogen), and testing compounds in $20 \mu \mathrm{L}$ of thermal shift buffer ( $25 \mathrm{mM}$ PIPES, pH 6.2, $150 \mathrm{mM} \mathrm{NaCl}, 1$ mM DTT) were heated from 25 to $95^{\circ} \mathrm{C}$ at $1 \%$ ramp rate. The change in the fluorescence intensities of SYPRO orange was monitored as a function of the temperature. Each reaction was repeated three times.

\section{Cell viability analysis}

BC cells $\left(1 \times 10^{3}-2 \times 10^{3}\right.$ cells per well) or lymphoma cell lines $(1 \times$ $10^{4}-2 \times 10^{4}$ cells per well) were placed in 96-well flat-bottomed microtiter plates in triplicate cultures, and incubated with indicated compounds. Sulforhodamine B (SRB) assay was used to detect breast cancer cells proliferation. And cell Counting Kit-8 (Vazyme, Nanjing, China) was used to detect lymphoma cells 
proliferation. The cell viability was expressed as the percentage of absorbance in cells with indicated treatments to that in the control cells. The dosages corresponding to the half-maximal growth inhibitory concentrations inhibition $\left(\mathrm{IC}_{50}\right)$ were calculated using SoftMax software.

Flow cytometric analysis of apoptosis and cell cycle

Cells were seeded at a density of $1 \times 10^{5}$ cells/well in 6-well culture plates and then transfected with miRNAs or treated with AZD9291 at the certain concentration for $48 \mathrm{~h}$ after transfection. Apoptosis was monitored using flow cytometric analysis with Annexin V-FITC Apoptosis Detection Kits (Vazyme) according to the manufacturer's instruction. To examine the cell cycle, fluorescence-activated cell sorting (FACS) analysis was performed. Cells were collected and fixed with $70 \%$ ethanol at $-20^{\circ} \mathrm{C}$ overnight. After centrifugation at $450 \times g$ for $5 \mathrm{~min}$, the cells were then incubated with RNaseA (200 mg/mL, Sangon Biotech, Shanghai, China) and stained with propidium iodide (PI) solution $(50 \mathrm{mg} / \mathrm{mL}$, Sigma-Aldrich, St. Louis, MO, USA) dissolved in PBS for 15 min at room temperature. The cells were examined using a FACS Calibur flow cytometer (BD Biosciences, San Jose, CA, USA). Cell Quest Pro software (BD Biosciences) was used to analyze the flow cytometric data.

\section{Cell migration and invasion assays}

Cells were seeded in 6-well culture plates, for the indicated treatment for $48 \mathrm{~h}$. Cells suspension containing $1 \times 10^{5}$ cells $/ \mathrm{mL}$ was prepared in serum-free medium and $100 \mu \mathrm{L}$ cells were added into the upper chamber inserts, $600 \mu \mathrm{L}$ medium supplemented with $10 \%(\mathrm{v} / \mathrm{v})$ FBS was added into the lower chambers (Corning, Kennebunk, ME, USA). Then migration was performed at $37^{\circ} \mathrm{C}$ for $12 \mathrm{~h}$. The invasive capacity of these transfected cells was determined by a similar experiment in which the Transwell chamber inserts were coated with matrigel matrix (Corning). Inserts were then fixed and stained with $0.1 \%$ crystal purple diluted in anhydrous ethanol. Non-migrated cells on the upper surface of the chamber were removed with cotton-tipped swabs. Dried cells were photographed and counted under a microscope by selecting three fields per filter ( $\times 20$ magnification).

\section{Wound healing assays}

MDA-MB-453 cells were seeded in 6-well plates. The surface of the plate was scraped using a $200 \mu \mathrm{L}$ pipette tip to generate a linear wound. Free cells were then removed with two washes with PBS, and cells were incubated in the $2 \mathrm{~mL}$ medium containing $2.5 \mu \mathrm{M}$ AZD9291 or $50 \mathrm{nM}$ miR-34a mimic or control. Cells were imaged using a time-lapse video microscopy system (Olympus, Tokyo, Japan) immediately.

\section{Colony formation assay}

Cells (500-1000 cells/well) with or without AZD9291 were plated in 6-well plates and incubated in RPMI (Gibco) with 10\% FBS at 37 ${ }^{\circ} \mathrm{C}$. Two weeks later, the cells were fixed with and stained with $0.1 \%$ crystal violet. The number of visible colonies was counted manually. All samples were assayed at least three independent experiments.

\section{Real-time qPCR assay}

Cells were plated in 6-well plates and incubated with AZD9291 for $48 \mathrm{~h}$, then the total RNA was extracted with Trizol, according to manufacturer's protocol (Invitrogen, Carlsbad, CA, USA). RNA was reverse-transcribed using HiScript II Q Select RT SuperMix for qPCR (R233-01 Vazyme). RT-qPCR was carried out according to the instructions for SYBR Green PCR master mix (6252, BioRad) by V7 Real-Time PCR system (Applied Biosystems) as follows: $95^{\circ} \mathrm{C}$ for $60 \mathrm{~s}$ followed by 40 cycles of amplification for $5 \mathrm{~s}$ at $95^{\circ} \mathrm{C}, 34 \mathrm{~s}$ at $60^{\circ} \mathrm{C}$. Relative gene expression was quantified using the $2^{-\triangle \Delta C T}$ method. The primers sequences used for PCR are listed in Table S2.
To examine miR-34 expression in cells, total RNA was extracted using the miRNeasy Mini Kit (Qiagen, Hilden, Germany) according to the manufacturer's recommended protocol and reversetranscribed to complementary DNA (cDNA) using the Mir- $X^{\mathrm{TM}}$ miRNA First-Strand Synthesis Kit (638315, Takara, Otsu, Japan). miR-34a expression was measured by qRT-PCR using the Mir- $\mathrm{X}^{\mathrm{TM}}$ miRNA qRT-PCR SYBR Kit (Takara) and normalized to U6 small nuclear RNA as an endogenous control. The $2^{-\Delta \Delta C T}$ method was used to calculate the relative level of miRNA expression. PCR was conducted by $95^{\circ} \mathrm{C}$ for $10 \mathrm{~s}$ followed by 40 cycles of amplification for $5 \mathrm{~s}$ at $95^{\circ} \mathrm{C}, 20 \mathrm{~s}$ at $60^{\circ} \mathrm{C}$.

Dual luciferase reporter assay

HEK293 cells were plated $\left(5 \times 10^{4}\right.$ cells/well) in a 24 -well plate for $24 \mathrm{~h}$ before transfection. The cells were transfected with the pmirGLO-EZH2-wt or pmirGLO-EZH2-mutant plasmids (200 ng) together with $50 \mathrm{nM}$ miR-34a mimic or control miRNAs. Luciferase activity was measured $48 \mathrm{~h}$ after transfection using the DualLuciferase Reporter Assay System, according to the manufacturer's instructions (Promega). For each sample, firefly luciferase activity was normalized to Renilla luciferase activity.

Statistical analysis

All values were analyzed using the two-tailed Student $t$-test, $P$-values $<0.05$ were considered statistically significant. Analyses were performed using GraphPad Prism (version 6.01) software. The log-rank test was employed to determine the association between miR-34a expression and overall survival, and the Kaplan-Meier method was utilized to generate survival curves.

\section{RESULTS}

AZD9291 disrupts the EZH2-EED interaction

NanoBRET ${ }^{\mathrm{TM}}$ Technology enables sensitive, reproducible detection of protein interactions in the natural cellular environment [13]. Here, we used EZH2 and EED proteins expressed in HEK293T cells to do protein-protein interaction (PPI) monitoring and screening studies that reflect true cellular physiology. An in-house compound bank, containing approximately 1300 known compounds, was screened using this method. Among them, AZD9291, with the concentration of $5 \mu \mathrm{M}$ disrupted the interaction between EZH2 and EED significantly, as well as astemizole, which has been reported as a small molecular inhibitor targeting the EZH2-EED interaction [14] (Fig. 1a). Other EGFR inhibitors, gefitinib and afatinib, and EZH2 enzymatic inhibitors EPZ-6438, GSK126, had no such effects in this assay (Fig. S1a). Then we performed Co-IP in HEK293T cell to identify if AZD9291 disrupted the binding of EZH2 and EED. As indicated in Fig. 1b, like EED226, AZD9291 treatment produced a clear decreased EED level when immunoprecipitation of EZH2 followed by immunoblotting with anti-EED antibody. Similar results were obtained using IP EED followed by immunoblotting with anti-EZH2 antibody (Fig. 1b). Ligand binding to a protein is known to increase its thermal stability, which is reflected by a shift between the thermal denaturation curves of the unliganded protein and the liganded protein. A fluorescence-based thermal shift assay is used to study thermal stabilization of proteins upon ligand binding [15]. Such assays have been used extensively on purified proteins in the drug discovery industry and in academia to detect protein interactions. In the assay, we found that AZD9291 did not dose-dependently shift the melting temperature (Tm) of EED until reaching a plateau (Fig. 1c), suggesting that AZD9291 did not directly bind to EED protein. Moreover, we found that AZD9291 might directly bind with EZH2, not EED or SUZ12, to increase its stability by CETSA, which method allows studies of target engagement of drug candidates in a cellular context [16]. On the Western blots, we found the presence of detected proteins at the lower test temperatures followed by its disappearance as the temperature 

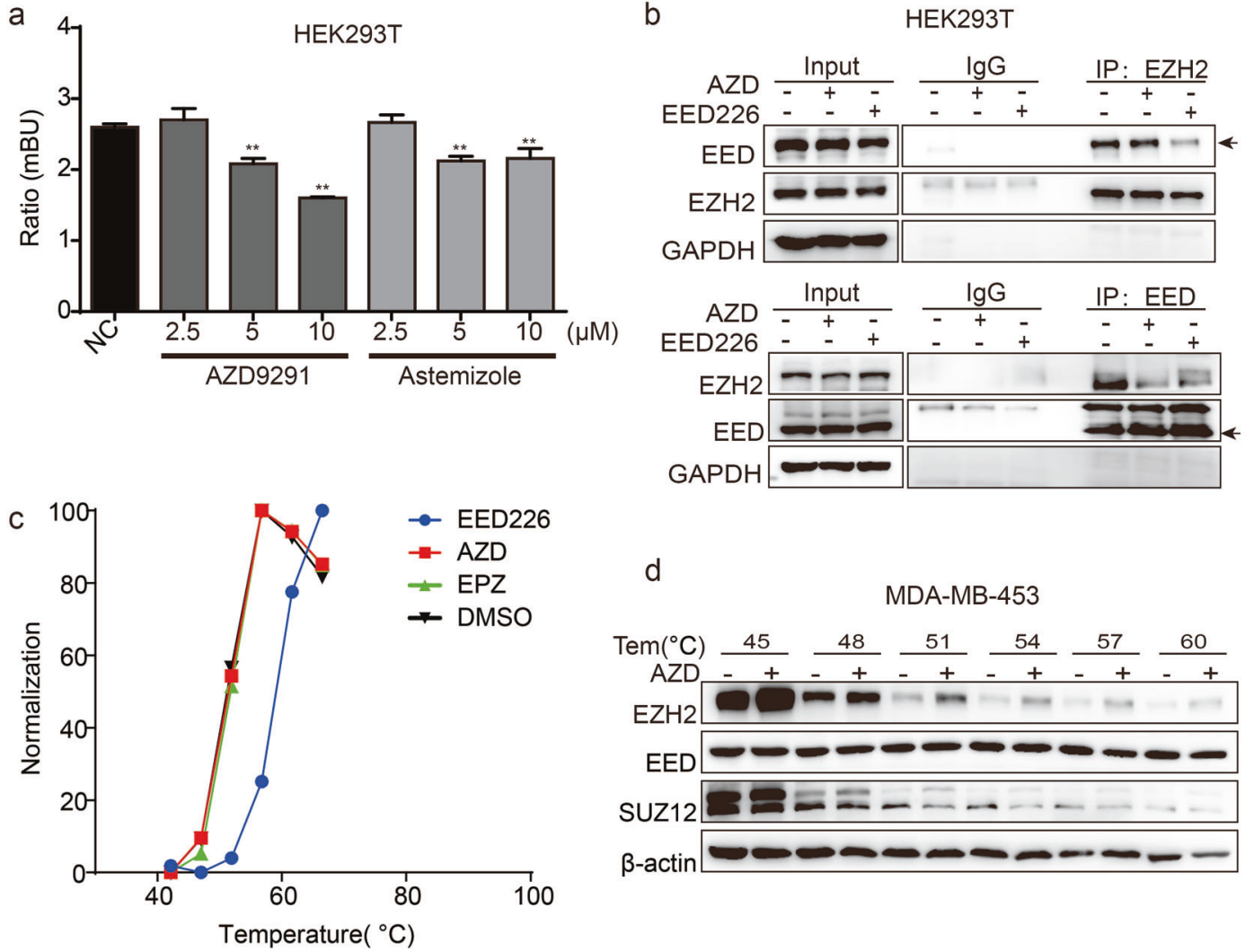

d

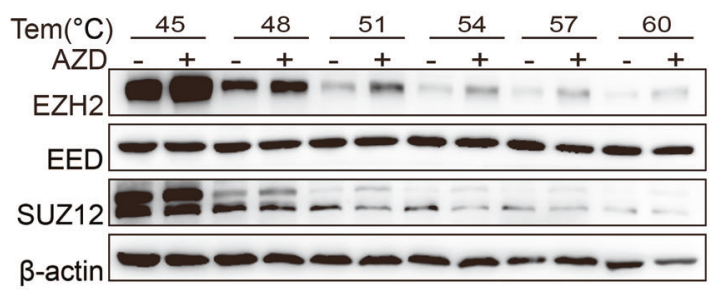

Fig. 1 AZD9291 disrupts the EZH2-EED interaction by binding to EZH2 directly. a NanoBRET assay. HEK293T cells were co-transfected with pFN31K-EZH2 NanoLuc and pFC14K-EED HaloTag vectors for about $20 \mathrm{~h}$ then treated with AZD or astemizole (the highest concentration was $10 \mu \mathrm{M}$, diluted by 2 times) overnight, donor emission $(460 \mathrm{~nm})$ and acceptor emission $(618 \mathrm{~nm})$ were measured using microplate reader and the ratio values with milliBRET units (mBU) were calculated as RawBRET $=618 \mathrm{~nm} \mathrm{Em} / 460 \mathrm{~nm} \mathrm{Em} \times 1000$. $n=3$ replicates. b HEK293T cell was exposed to AZD $(2.5 \mu \mathrm{M})$ or EED226 $(2.5 \mu \mathrm{M})$ for $48 \mathrm{~h}$. The cell lysates were incubated with anti-EZH2 or immunoglobulin G (lgG) antibodies. Immunoprecipitation was immunoblotted with the indicated antibodies. c Each reaction solution containing $2 \mu M$ EED, $5 \times$ SYPRO orange, and testing compounds $(25 \mu \mathrm{M})$ in $20 \mu \mathrm{L}$ thermal shift buffer. The melting temperature $(\mathrm{Tm})$ was calculated by the Boltzmann fitting method. $\mathbf{d}$ MDAMB-453 cells were pre-treated with $10 \mu \mathrm{M}$ MG132 for $1 \mathrm{~h}$ before treatment with the $20 \mu \mathrm{M}$ AZD for $4 \mathrm{~h}$. Cell lysates were used for SDS PAGE and Western blotting. All error bars represent mean \pm SD analyzed by unpaired two-tailed $t$ test. ${ }^{* *} P<0.01$. AZD, AZD9291. See also Figure S1

increased. The encouraging thing was that only $\mathrm{EZH} 2$ protein appeared at higher temperature after incubation with AZD9291 (Figs. $1 \mathrm{~d}$ and $\mathrm{S} 1 \mathrm{~b}$ ). Taken together, these results strongly suggested that AZD9291 selectively bound to EZH2 and disrupted the EZH2-EED interaction.

AZD9291 selectively inhibits H3K27 methylation and exhibits antitumor activity

Previous studies emphasized the importance of the EZH2-EED interaction for the integrity of the PRC2 complex [17], so the dissociation of the EZH2-EED complex by AZD9291 was explored in cancer cells. Diffuse large B-cell lymphoma cell lines (DLBCL) U2932 and DB, and BC cell lines MDA-MB-453, T47D were exposed to AZD9291 or an enzymatic inhibitor, EPZ-6438 for $72 \mathrm{~h}$. As expected, AZD9291 dose-dependently decreased the EZH2, EED, SUZ12 and H3K27me3 levels in all tested BC and DLBCL cell lines. By contrast, EPZ-6438, which competes for SAM, only inhibited H3K27 methylation, and had no effect on EZH2, EED and SUZ12 expression. In addition, the other histone $\mathrm{H} 3$ methylation modifications were unaffected by AZD9291 and EPZ-6438, highlighting the exquisite specificity of AZD9291 for PRC2 inhibition (Fig. 2a). To better understand the cellular effect of AZD9291, a few genes which were reported as target of PRC2 were selected for validation by $\mathrm{qRT}-\mathrm{PCR}$. The changes of gene expression induced by AZD9291 were similar to those induced by EPZ-6438 in BC and lymphoma cell lines. (Fig. 2b and S2a). The data strongly suggested that AZD9291 inhibited PRC2 complex activity.
Then we explored the antitumor activity of AZD9291. As shown in Fig. 2C, AZD9291 inhibited the proliferation of BC cells and lymphoma cells carrying mutant form of EZH2 or wild-type EZH2 at micromolar concentration $(0.015-19.6 \mu \mathrm{M})$. We also found that after a long time treatment, AZD9291 decreased the colony formation number of BC cells (Fig. 2d). We then examined the effects of AZD9291 on cell cycle distribution and apoptotic ratio of BC cells MDA-MB-453 and T47D, and lymphoma cells U2932 and DB. Compared with control, there was an accumulation of cell population in $G_{0} / G_{1}$ phase and no significant effect on apoptotic ratio was observed after treatment with AZD9291 (Fig. 2e and $\mathrm{S} 2 \mathrm{~b})$. EZH2 has been demonstrated clearly that it is associated with tumor invasion and metastasis [18, 19]. To determine whether AZD9291 inhibits cell migration, we performed wound healing assay and transwell migration assay. As shown in Fig. $2 \mathrm{f}-\mathrm{g}$, the presence of AZD9291 significantly suppressed cell migration and invasion of MDA-MB-453 cells.

Considering that AZD9291 is an EGFR inhibitor, we further examined whether the effect of AZD9291 on the stability of PRC2 dependents on EGFR expression. We found that depletion of EGFR by a specific siRNA (siEGFR) did not affect EZH2 expression. Further, AZD9291 still decreased EZH2 and H3K27me3 expression in EGFRdepleted MDA-MB-453 cells (Fig. S2c). Meanwhile, when EGF factor was used to activate the EGFR pathway in advance, AZD9291 also decreased the expression of EZH2 (Fig. S2d). All these data indicated that AZD9291 decreased the protein level of EZH2, inhibited PRC2 activity, independent of the expression of EGFR. 

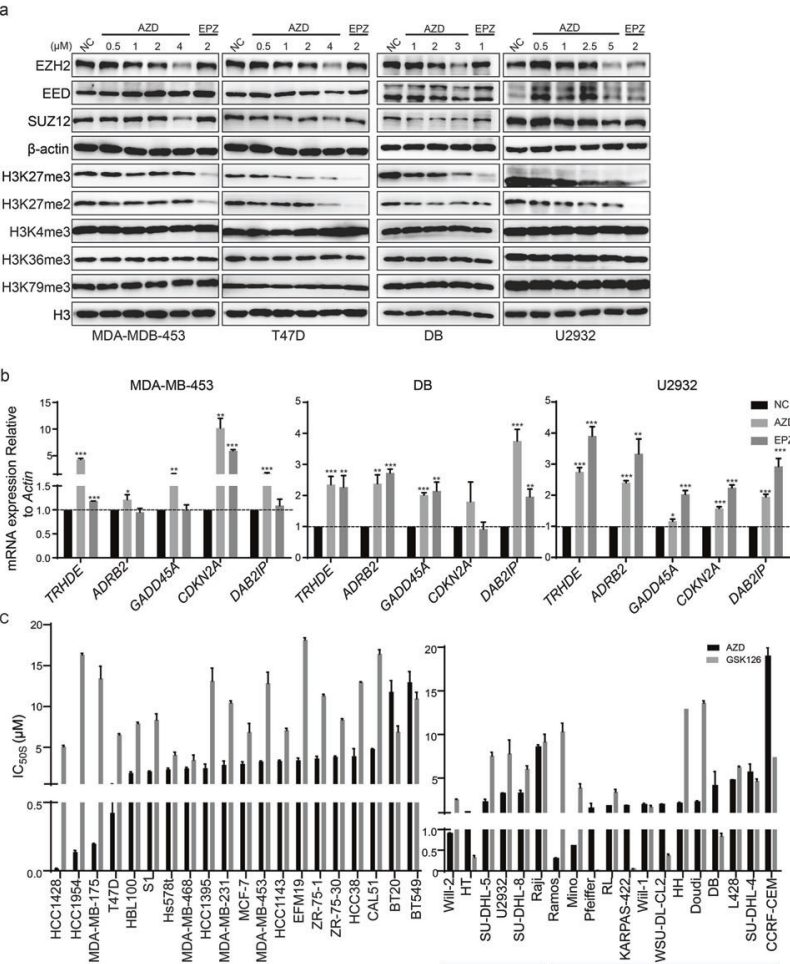
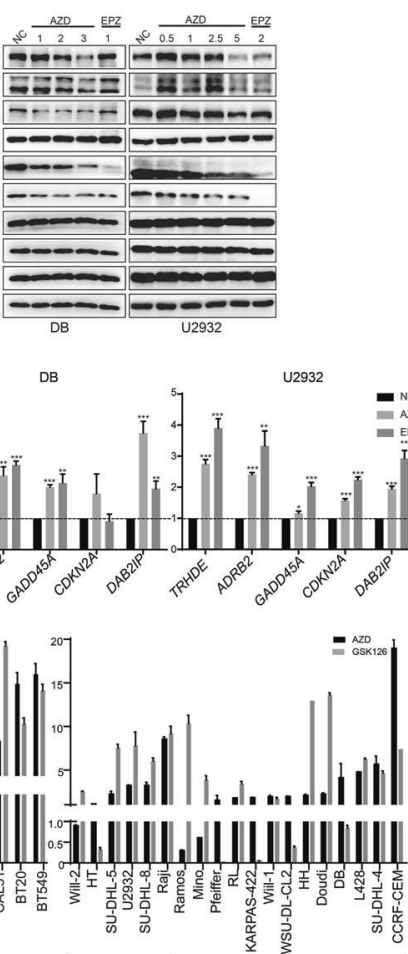

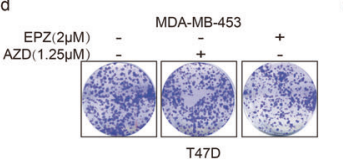

$\operatorname{EPZ}(2 \mu \mathrm{M})$
$\operatorname{AZD}(0.625 \mu \mathrm{M})$
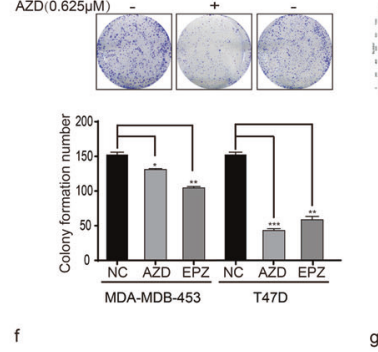

g

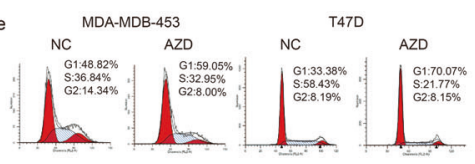

NC U2932 AZD

NC

DB AZD
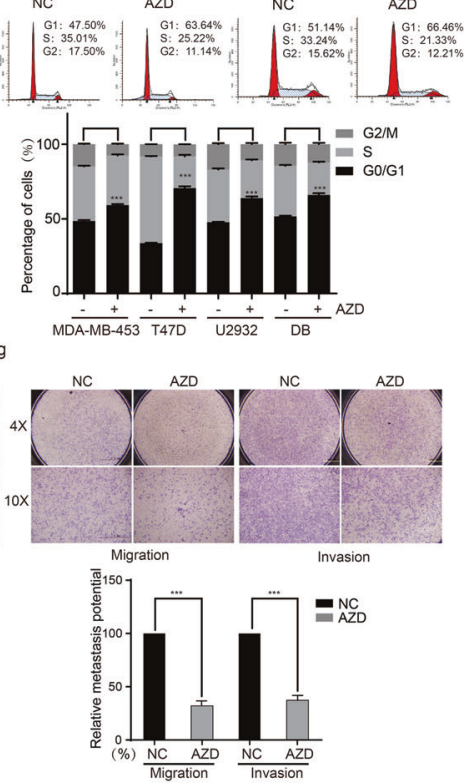

Fig. 2 AZD9291 selectively inhibits H3K27 methylation and exhibits anti-tumor activity. a EZH2, EED, SUZ12 protein, H3K27me3 and other histone lysine methylation or acetylation marks levels in MDA-MB-453 and T47D breast cancer cells and DLBCL cell lines U2932 (EZH2 WT type) and DB (EZH2 mutant type) were shown by following treatment with the indicated concentrations of AZD or EPZ for 72 h. The $\beta$-actin and total histone H3 were shown as a loading control. b Cells were treated with the AZD $(2.5 \mu \mathrm{M})$ or EPZ $(2.5 \mu \mathrm{M})$ for $48 \mathrm{~h}$. The mRNA level of PRC2 targeted genes (fold change) was obtained as normalized by that of the DMSO-treated group. $n=3$ replicates. c Cells were treated with AZD or GSK1 26 at gradient concentrations for 7 days and IC ${ }_{50 \text { s }}$ measured using SRB assay or CCK-8 assay. $\mathbf{d}$ Colony-forming assay. Cells were treated with AZD or EPZ for about 2 weeks and cells growth changes were measured by counting colony numbers. $n=3$ replicates. e The cell cycle was examined by flow cytometry after treated with AZD 2-2.5 $\mu \mathrm{M}$ for $48 \mathrm{~h}$. $\mathbf{f}, \mathbf{g}$ The influence of AZD on cancer cells metastasis was detected by wound-healing experiment (f), Transwell experiment and Matrigel invasion experiment (g) to evaluate the mobile ability of cancer cells. Scale bar, $2 \mathrm{~mm}$. All error bars represent mean \pm SD analyzed by unpaired two-tailed $t$ test. ${ }^{*} P<0.05,{ }^{* *} P<0.01,{ }^{* * *} P<0.001$. AZD, AZD9291. EPZ, EPZ6438. See also Figure S2

\section{AZD9291 decreases EZH2 expression}

Previous studies emphasized the dissociation of the EZH2-EED complex may impair the stability of EZH2 in cells. So we detected AZD9291's effect on the stability of EZH2. Cycloheximide (CHX) is always used to inhibit protein de novo synthesis. As expected, in the cells treated with CHX and AZD9291 together, the half-life of EZH2, EED and SUZ12 was much shorter compared to the treatment with AZD9291 $5 \mu \mathrm{M}$ alone (Fig. 3a). Moreover, we found that treatment with AZD9291 increased the EZH2 ubiquitination in MDA-MB-435 cells (Fig. 3b). All these data indicated that AZD9291 attenuated EZH2 stability.

To our surprise, quantitative real-time $P C R$ showed that AZD9291 treatment significantly decreased the mRNA of EZH2 in $B C$ and DLBCL cell lines (Fig. 3c). Several studies have indicated that there is a phenotypic discrepancy between the genetic abrogation and enzymatic inhibition of $\mathrm{EZH} 2$, and have further highlighted the dominant contributions of the non-catalytic functions in several cancer models [20-22]. Then mRNA levels of the BRIC5, ARL6IP, TACC3, CEP76, CENPK and CHEK1 genes, which are activated by EZH2 independent of PRC2 were analyzed after treatment with AZD9291. All these genes expressions in $B C$ and $D L B C L$ cells significantly declined as expected (Fig. 3d). Above all, AZD9291 not only disturbed the interaction of EZH2 and EED, attenuated EZH2 stability, but also decreased the mRNA level of EZH2 and its activated genes.

AZD9291 attenuates EZH2 mRNA via miR-34a

MicroRNAs (miRNAs) have been shown to negatively regulate gene expression by binding to complementary sequence sites in the $3^{\prime}$-untranslated regions of the mRNAs of protein-coding genes, thereby degrading or blocking the translation of these mRNAs [23]. This prompted us to investigate whether miRNAs contribute to EZH2's dysregulation by AZD9291. We performed small RNAsequencing to examine the changes of miRNAs expression in AZD9291-treated MDA-MB-453 cells. Then 38 significantly upregulated and 50 downregulated miRNAs were identified (Fig. $4 a$ and Supplement Table S1). Among these upregulated miRNAs, miR-34a (miR-34a-5p) had attracted our interest, because of its ability to modulate myriad of oncogenic functions in different kinds of cancer [24-26]. Firstly, we determined the clinical significance and prognostic value of miR-34a expression by analyzing a cohort from the Kaplan-Meier-plotter (http://kmplot. com/analysis/). When METABRIC $(n=1262)$ dataset selected, miR34 a levels were stratified by the median level, lower levels of miR34a were associated with poor overall survival for patients with BC (hazard ratio $=0.8,95 \%$ confidence interval $=0.65,0.98$; $P[$ logrank test $]=0.028$ ). Kaplan-Meier plots were generated for the median cutoff (Fig. 4b). When TCGA $(n=1077)$ dataset selected, identified 97 TNBC patients, lower level of miR-34a was associated with poor overall survival (hazard ratio $=0.24,95 \%$ confidence interval $=0.07,0.85$; $P[$ log-rank test $]=0.017$ ) (Fig. 4c), as same result in 996 ER-positive $\mathrm{BC}$ patients (hazard ratio $=0.75,95 \%$ confidence interval $=0.59,0.94$; $P[$ log-rank test $]=0.012$ ) (Fig. $4 d$ ).

Then qRT-PCR analysis was used to substantiate the small RNAsequencing result. As expected, the relative level of miR-34a was significantly upregulated in MDA-MB-453, U2932, and Pfeiffer cells after treatment with AZD9291 (Fig. 4e). We also observed that 

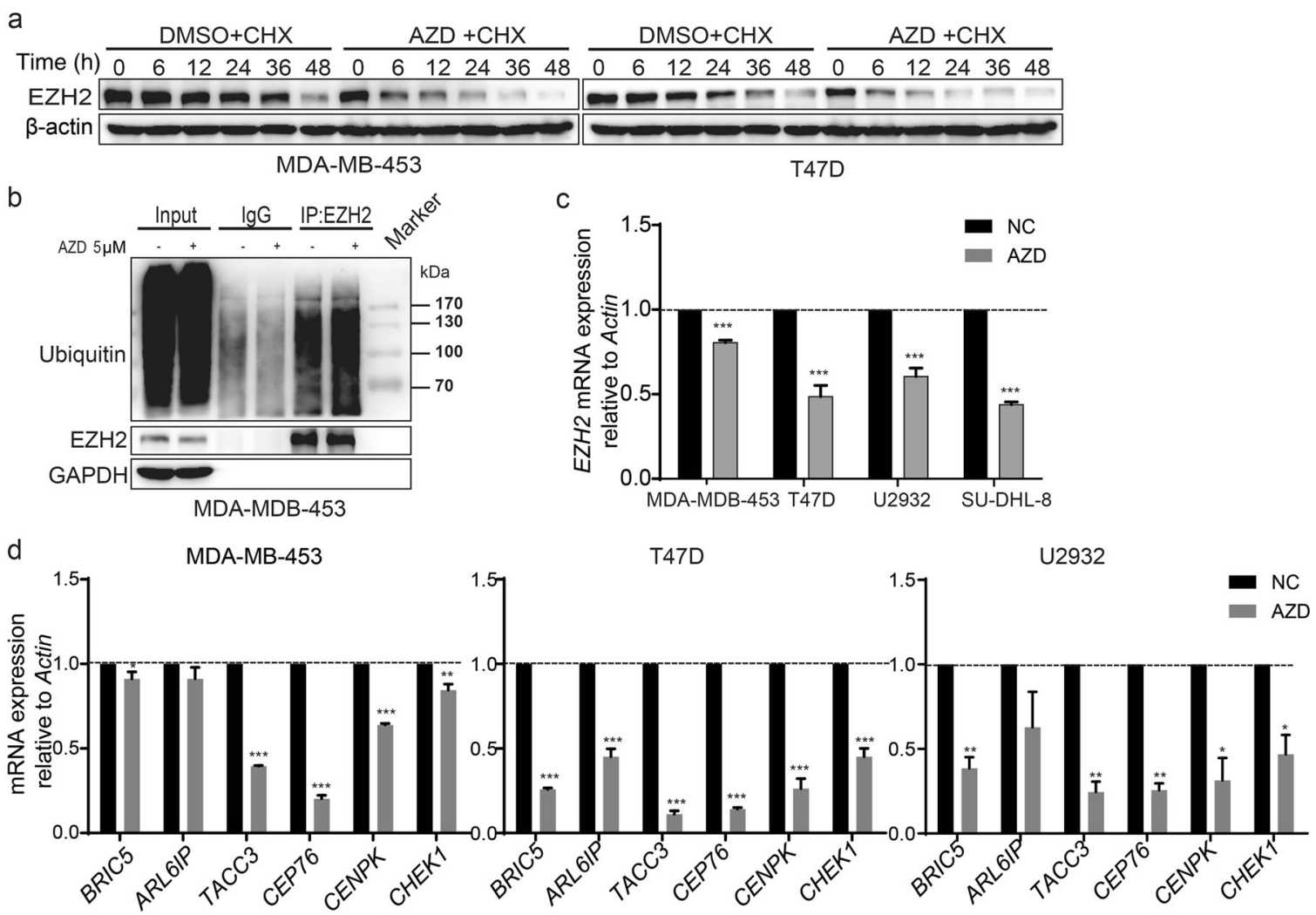

Fig. 3 AZD9291 decreases EZH2 expression and inhibits genes expression which EZH2 activated. $\mathbf{a}$, b Cells were harvested for detection of protein level by Western blotting. Cells were treated with cycloheximide $(100 \mu \mathrm{M})$ combined with vehicle (DMSO) or AZD (5 $\mu \mathrm{M})$ for the indicated times (a). After treatment with $5 \mu \mathrm{M}$ AZD for $48 \mathrm{~h}, \mathrm{MDA}-\mathrm{MB}-453$ cells were lysed and subjected to the EZH2 ubiquitination assay (b). c, d Cells were treated with AZD $2.5 \mu \mathrm{M}$ for $48 \mathrm{~h}$ then samples were subjected to qRT-PCR assay. The mRNA expression of EZH2 (c) and EZH2 activated genes (d) were tested. Error bars represent mean $\pm \mathrm{SD}$ analyzed by unpaired two-tailed $t$ test. ${ }^{*} P<0.05,{ }^{* *} P<0.01,{ }^{* * *} P<0.001$. AZD, AZD9291. CHX, Cycloheximide

EZH2 mRNA was reduced in tested breast cancer cells transiently transfected with miR-34a mimics (Fig. 4f). The decreased EZH2 protein level and impaired H3K27me3 were confirmed by Western blot analysis (Fig. 4g). In order to identify the potential targets of miR-34a that might contribute to the regulation of $E Z H 2$, we performed an unbiased computational screen by multiple prediction algorithms (Targetscan, PicTar, and miRanda). We did not find the binding sites for miR-34a "seed sequence" in the 3' UTR of EZH2. However, we found that there was a non-classical binding site in the EZH2 3'UTR (Fig. 4h). Pmir-GLO Dual-Luciferase miRNA target expression vector bearing the EZH2 wild type (wt) or mutated (mutant) full-length 3'UTRs were transfected into HEK293 cell, after treatment with miR-34a for $48 \mathrm{~h}$, the luciferase activity was only reduced in WT $3^{\prime}$ UTR groups but not in the mutated groups (Fig. 4i). It suggested that miR-34a might interact with the region at $172-178$ of EZH2 $3^{\prime}$ UTR to decrease its mRNA, which needto be further confirmed.

We then determined whether the modulation of miR-34a by AZD9291 is important for the suppressed expression of EZH2. If the increased expression of miR-34a is crucial for the downregulation of EZH2, we would expect miR-34a could mimic AZD9291's effect on EZH2. Indeed, miR-34a had the same effects with AZD9291 on cell cycle (Fig. 5a), apoptosis (Fig. 5b), and metastasis (Fig. 5c, d). In addition, miR-34a downregulated the mRNA expression of $\mathrm{EZH} 2$ activated genes and upregulated EZH2 inhibited genes (Fig. 5e, f). Furthermore, infection with the inhibitor of miR-34a (anti-miR-34a) partially restored the protein level of EZH2 and H3K27me3 in AZD9291treated cells (Fig. 5g). In dual-luciferase test, AZD9291 also decreased EZH2 3'UTR expression like miR-34a did (Fig. 5h). The above results suggested that the attenuating effect of AZD9291 on EZH2 protein was partially dependent on the upregulation of miR-34a expression.

\section{DISCUSSION}

Epigenetic regulators are emerging as a new class of drug targets, $\mathrm{EZH} 2$ is one of the hot targets. Yet clinical benefits of EZH2 enzymatic inhibitors remain unsatisfactory and limited to certain hematological malignancies. Whereas other types of EZH2 inhibitors remain to be optimized, such EZH2-EED interaction disruptors are still in the preclinical stage [10] [NCT02900651]. In this study, we showed that the AZD9291, a potent and selective EGFR inhibitor, disturbed the interaction of EZH2-EED, leading to impairment of PRC2 activity, promoting EZH2 degradation, which was independent of EGFR expression. Further, AZD9291 upregulated the expression of miR-34a, which targeted EZH2 3'UTR and decreased its mRNA and protein level (Fig. 5i). All these data supported that AZD9291 could serve as a lead compound for further development.

$\mathrm{EZH} 2$ is aberrantly overexpressed in various malignant tumors, such as prostate cancer, breast cancer, and ovarian cancer [27, 28]. The role of $\mathrm{EZH} 2$ in cancer progression is highlighted and unraveled. Accumulating evidence indicates that beyond playing its role in a PRC2-dependent manner as a histone modifier, EZH2 also functions both as a transcriptional suppressor and a transcriptional co-activator, not depending on H3K27me3 but on the different cellular contexts [29]. Through modulating critical gene expression, EZH2 promotes cell survival, proliferation, invasion [28, 30], and drug resistance of cancer cells [31, 32]. Thus, EZH2 is a potential target for cancer therapy, and 
MDA-MB-453(AZD vs DMSO)

\begin{tabular}{cccc}
\hline miRNA_ID & log2(fold change) & $P$-value & Significance-Lab \\
\hline hsa-miR-34a-5p & 7.213 & 0.00021796 & $* *$ \\
hsa-miR-4284 & 7.0609 & 0.000390825 & $* *$ \\
hsa-miR-6724-5p & 6.8911 & 0.000726851 & $* *$ \\
hsa-miR-182-3p & 6.6984 & 0.001408973 & $* *$ \\
hsa-miR-556-5p & 6.6984 & 0.001408973 & $* *$ \\
hsa-miR-5094 & 6.6984 & 0.001408973 & $* *$ \\
hsa-miR-3157-3p & 6.5914 & 0.001996045 & $* *$ \\
hsa-miR-4435 & 6.5914 & 0.001996045 & $* *$ \\
hsa-miR-1827 & 6.5914 & 0.001996045 & $* *$ \\
hsa-miR-6787-3p & 6.5914 & 0.001996045 & $* *$ \\
\hline
\end{tabular}

$\mathrm{b}$ 

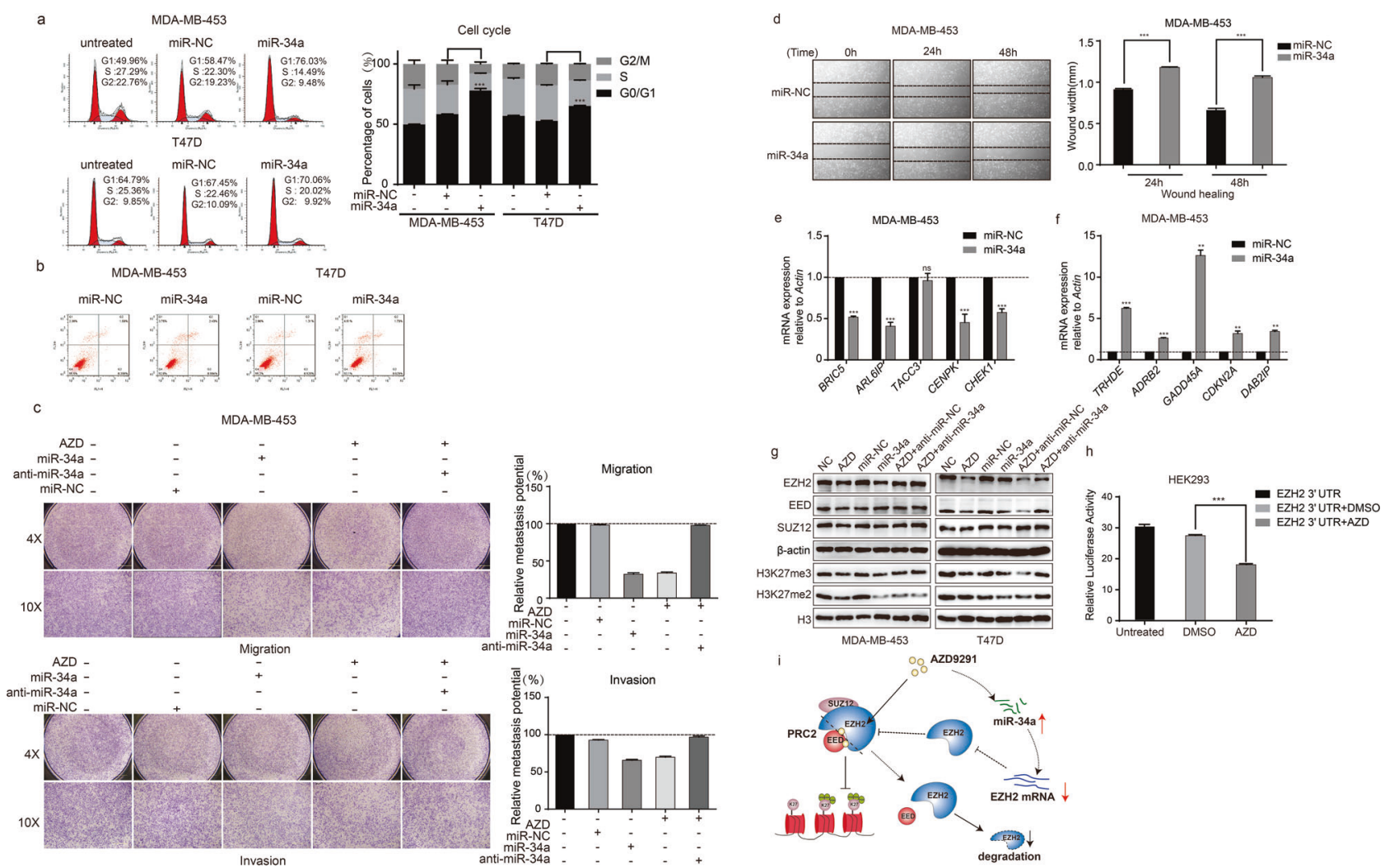

Fig. 5 miR-34a has the same effects with AZD9291. a, b Cells were transfected with the miR-34a or miR-NC $100 \mathrm{nM}$ for $48 \mathrm{~h}$, then the influences of miR-34a on the cell cycle (a) and apoptosis (b) were examined by flow cytometry. $\mathbf{c}$, $\mathbf{d}$ The influences of AZD and miR-34a on cancer cell metastasis. AZD $2.5 \mu \mathrm{M}$ or miR-34a $100 \mathrm{nM}$ treated MDA-MB-453 cells for $48 \mathrm{~h}$ were subjected to transwell experiment and matrigel invasion experiment (c) and wound-healing experiment (d). Scale bar, $2 \mathrm{~mm}$. e, f qRT-PCR assay. MDA-MB-453 cells were treated with miR-34a or AZD $2.5 \mu \mathrm{M}$ for $48 \mathrm{~h}$ then samples were subjected to qRT-PCR assay. The mRNA level of EZH2 activated genes (e) and inhibited genes (f) were tested. g Western blotting. Cells were harvested after transfected with $100 \mathrm{nM}$ miR-34a mimics or anti-miR-34a alone or combined with AZD $2.5 \mu \mathrm{M}$ for $48 \mathrm{~h}$. h Dual-luciferase assay. HEK293 cells were treated with AZD $2.5 \mu \mathrm{M}$ for $48 \mathrm{~h}$, then the luciferase activity was tested. $\mathbf{i}$ A hypothetical model illustrating the silencing mechanism of AZD on PRC2 in cancer cells. All error bars represent mean \pm SD analyzed by unpaired two-tailed $t$ test. ${ }^{* *} P<0.01,{ }^{* * *} P<0.001$. AZD, AZD9291

compounds targeting the regulation of $\mathrm{EZH} 2$ expression may also be another approach with efficacy against various neoplastic diseases. 3-Deazaadenosine A (DZNep) is a non-specific EZH2 inhibitor which decreases the protein level of EZH2 and the H3K27me3 marks, thereby exhibiting antitumor effects in various malignancies. However, DZNep was reported to have efficacy but also toxicity in animal models [33]. Here, we proved that AZD9291 could both disrupt PRC2 complex and decreased EZH2 mRNA expression, which lead to suppressing the tumor growth.

miRNAs are small noncoding RNAs that typically repress the expression of target genes through mRNA degradation or inhibition of translation [34]. EZH2 has been considered to be regulated by miRNAs to promote disease progression. Some miRNAs (e.g., miR-126, miR-138, miR-32, miR-26a, miR-506, miR137 , and miR-101) are reported to directly target EZH2 in different types of cancer and display different functions [35-39]. For example, miR-126 and miR-138 directly target EZH2 transcript and increase the sensitivity of osteosarcoma cells and gastric cancer cells to chemotherapy. In this study, we performed small RNAsequencing and identified that the down-regulation of EZH2 mRNA was caused by miR-34a. miR-34a and its downstream targets have been found to influence cancer stem cells proliferation, metastasis, and chemoresistance of cancers, so that small molecules having a similar function as miR-34a are often regarded as better candidates for cancer treatment [40-43]. MiR-34a expression is reported to be inactivated by aberrant DNA methylation and this is implicated in tumor suppression [44]. In addition, it has been reported that EZH2 decreases the miR-34a promoter activity though physically interacted with HOTAIR and represses its expression [45]. Treatment with the EZH2 enzymatic inhibitor, GSK126, or the DNA methylation inhibitor, 5-Aza-2'deoxycytidine, partially restored miR-34a levels [46]. However, in our study, we found that miR-34a was upregulated by AZD9291 in DLBCL cells and breast cancer cells. We also proved that there was a non-classical binding site in the EZH2 $3^{\prime}$ UTR for miR-34a, and suggested that EZH2 mRNA may be a direct target of miR34a with non-canonical base pairing way, which may give us some hints about miRNA complex and flexible targeting rules. We also found that low expression of miR-34a was associated with poor overall survival in BC patients, especially in TNBC patients. We demonstrated that upregulation of miR-34a inhibited tumor cells proliferation and invasion by decreasing EZH2. These indicated that miR-34a might be a useful biomarker to identify possible responders, which expand the translation avenue for $\mathrm{EZH} 2$ inhibitors and combination therapy in cancer treatments.

AZD9291, as an irreversible and mutant-selective EGFR inhibitor has shown extinguish effect for the treatment of NSCLC patients. In this study, it was characterized as a PRC2 inhibitor targeting both $\mathrm{EZH} 2$ enzymatic function and protein expression for the first time. These findings provide new molecular skeleton for PRC2 inhibitors, and a better therapeutic option especially in most solid tumors and big subset hematological malignancies, which EZH2 is overexpressed in a wild-type form. 


\section{AUTHOR CONTRIBUTIONS}

KLZ, QQS, and YMS performed experiments and analyzed data. KLZ, YFF, JD, and YC designed the research, drafted and edited the manuscript. YC and JD supervised the research.

\section{ADDITIONAL INFORMATION}

The online version of this article (https://doi.org/10.1038/s41401-019-0248-2) contains supplementary material, which is available to authorized users.

Conflict of interest: The authors declare no competing interests.

\section{REFERENCES}

1. Lewis EB. A gene complex controlling segmentation in Drosophila. Nature. 1978;276:565-70.

2. Ntziachristos $P$, Tsirigos A, Van Vlierberghe $P$, Nedjic J, Trimarchi T, Flaherty MS, et al. Genetic inactivation of the polycomb repressive complex 2 in T cell acute lymphoblastic leukemia. Nat Med. 2012;18:298-301.

3. De Raedt T, Beert E, Pasmant E, Luscan A, Brems H, Ortonne N, et al. PRC2 loss amplifies Ras-driven transcription and confers sensitivity to BRD4-based therapies. Nature. 2014;514:247-51

4. Nikoloski G, Langemeijer SM, Kuiper RP, Knops R, Massop M, Tonnissen ER, et al. Somatic mutations of the histone methyltransferase gene EZH2 in myelodysplastic syndromes. Nat Genet. 2010;42:665-7.

5. Morin RD, Johnson NA, Severson TM, Mungall AJ, An J, Goya R, et al. Somatic mutations altering EZH2 (Tyr641) in follicular and diffuse large B-cell lymphomas of germinal-center origin. Nat Genet. 2010;42:181-5.

6. Bodor C, O'Riain C, Wrench D, Matthews J, lyengar S, Tayyib H, et al. EZH2 Y641 mutations in follicular lymphoma. Leukemia. 2011;25:726-9.

7. McCabe MT, Graves AP, Ganji G, Diaz E, Halsey WS, Jiang Y, et al. Mutation of A677 in histone methyltransferase EZH2 in human B-cell lymphoma promotes hypertrimethylation of histone H3 on lysine 27 (H3K27). Proc Natl Acad Sci USA. 2012;109:2989-94.

8. Di Croce L, Helin K. Transcriptional regulation by Polycomb group proteins. Nat Struct Mol Biol. 2013;20:1147-55.

9. Kim W, Bird GH, Neff T, Guo G, Kerenyi MA, Walensky LD, et al. Targeted disruption of the EZH2-EED complex inhibits EZH2-dependent cancer. Nat Chem Biol. 2013;9:643-50.

10. Qi W, Zhao K, Gu J, Huang Y, Wang Y, Zhang $H$, et al. An allosteric PRC2 inhibitor targeting the H3K27me3 binding pocket of EED. Nat Chem Biol. 2017;13:381-8.

11. He $Y$, Selvaraju S, Curtin ML, Jakob CG, Zhu $H$, Comess KM, et al. The EED protein-protein interaction inhibitor A-395 inactivates the PRC2 complex. Nat Chem Biol. 2017;13:389-95.

12. Lu X, Yu L, Zhang Z, Ren X, Smaill JB, Ding K. Targeting EGFRL858R/T790M and EGFRL858R/T790M/C797S resistance mutations in NSCLC: current developments in medicinal chemistry. Med Res Rev. 2018;38:1550-81.

13. Machleidt T, Woodroofe CC, Schwinn MK, Mendez J, Robers MB, Zimmerman K, et al. NanoBRET-a novel BRET platform for the analysis of protein-protein interactions. ACS Chem Biol. 2015;10:1797-804.

14. Kong $X$, Chen $L$, Jiao $L$, Jiang $X$, Lian F, Lu J, et al. Astemizole arrests the proliferation of cancer cells by disrupting the EZH2-EED interaction of polycomb repressive complex 2. J Med Chem. 2014;57:9512-21.

15. Lucet IS, Murphy JM. Characterization of ligand binding to pseudokinases using a thermal shift assay. Methods Mol Biol. 2017;1636:91-104.

16. Jafari $R$, Almqvist $H$, Axelsson $H$, Ignatushchenko $M$, Lundback $T$, Nordlund $P$, et al. The cellular thermal shift assay for evaluating drug target interactions in cells. Nat Protoc. 2014;9:2100-22.

17. Denisenko OS, Suzuki M, Bomsztyk H. Point mutations in the WD40 domain of Eed block its interaction with Ezh2. Mol Cell Biol. 1998;18:5634-42.

18. Zingg D, Debbache J, Schaefer SM, Tuncer E, Frommel SC, Cheng P, et al. The epigenetic modifier EZH2 controls melanoma growth and metastasis through silencing of distinct tumour suppressors. Nat Commun. 2015;6:6051.

19. Li Z, Hou P, Fan D, Dong M, Ma M, Li H, et al. The degradation of EZH2 mediated by IncRNA ANCR attenuated the invasion and metastasis of breast cancer. Cell Death Differ. 2017:24:59-71.

20. Kim KH, Kim W, Howard TP, Vazquez F, Tsherniak A, Wu JN, et al. SWI/SNF-mutant cancers depend on catalytic and non-catalytic activity of EZH2. Nat Med. 2015;21:1491-6.

21. Lee ST, Li Z, Wu Z, Aau M, Guan P, Karuturi RK, et al. Context-specific regulation of NF-kappaB target gene expression by $\mathrm{EZH} 2$ in breast cancers. Mol Cell. 2011;43:798-810.
22. Xu K, Wu ZJ, Groner AC, He HH, Cai C, Lis RT, et al. EZH2 oncogenic activity in castration-resistant prostate cancer cells is Polycomb-independent. Science. 2012;338:1465-9.

23. Ambros V. The functions of animal microRNAs. Nature. 2004;431:350-5.

24. Ito $Y$, Inoue A, Seers $T$, Hato $Y$, Igarashi A, Toyama $T$, et al. Identification of targets of tumor suppressor microRNA-34a using a reporter library system. Proc Natl Acad Sci USA. 2017;114:3927-32.

25. Shi Y, Liu C, Liu X, Tang DG, Wang J. The microRNA miR-34a inhibits non-small cell lung cancer (NSCLC) growth and the CD44hi stem-like NSCLC cells. PLoS One 2014;9:e90022.

26. Adams BD, Parsons C, Slack FJ. The tumor-suppressive and potential therapeutic functions of miR-34a in epithelial carcinomas. Expert Opin Ther Targets. 2016;20:737-53.

27. Cebria F, Kobayashi C, Umesono Y, Nakazawa M, Mineta K, Ikeo K, et al. The polycomb group protein $\mathrm{EZH} 2$ is involved in progression of prostate cancer Nature. 2002;419:624-9.

28. Bachmann IM, Halvorsen OJ, Collett K, Stefansson IM, Straume O, Haukaas SA et al. EZH2 expression is associated with high proliferation rate and aggressive tumor subgroups in cutaneous melanoma and cancers of the endometrium, prostate, and breast. J Clin Oncol. 2006;24:268-73.

29. Gan L, Yang Y, Li Q, Feng Y, Liu T, Guo W. Epigenetic regulation of cancer progression by EZH2: from biological insights to therapeutic potential. Biomark Res. 2018;6:10.

30. Bracken AP. EZH2 is downstream of the pRB-E2F pathway, essential for proliferation and amplified in cancer. EMBO J. 2003;22:5323-35.

31. Gardner EE, Lok BH, Schneeberger VE, Desmeules P, Miles LA, Arnold PK, et al. Chemosensitive relapse in small cell lung cancer proceeds through an EZH2SLFN11 axis. Cancer Cell. 2017;31:286-99.

32. Fan TY, Wang H, Xiang P, Liu YW, Li HZ, Lei BX, et al. Inhibition of EZH2 reverses chemotherapeutic drug TMZ chemosensitivity in glioblastoma. Int J Clin Exp Pathol. 2014;7:6662-70.

33. Mayr C, Wagner A, Stoecklinger A, Jakab M, Illig R, Berr F, et al. 3-Deazaneplanocin A may directly target putative cancer stem cells in biliary tract cancer. Anticancer Res. 2015;35:4697-705.

34. Fabian MR, Sonenberg N, Filipowicz W. Regulation of mRNA translation and stability by microRNAs. Annu Rev Biochem. 2010;79:351-79.

35. Wang $P$, Li Z, Liu H, Zhou D, Fu A, Zhang E. MicroRNA-126 increases chemosensitivity in drug-resistant gastric cancer cells by targeting EZH2. Biochem Biophys Res Commun. 2016;479:91-6.

36. Zhu Z, Tang J, Wang J, Duan G, Zhou L, Zhou X. MiR-138 acts as a tumor suppressor by targeting EZH2 and enhances cisplatin-induced apoptosis in osteosarcoma cells. PLoS One 2016;11:e0150026.

37. Zhang $\mathrm{D}, \mathrm{Ni} Z, \mathrm{Xu} X$, Xiao J. MiR-32 functions as a tumor suppressor and directly targets EZH2 in human oral squamous cell carcinoma. Med Sci Monit. 2014;20:2527-35.

38. Zhang T, Qian H, Hu C, Lu H, Li JB, Wu YF, et al. MiR-26a mediates ultraviolet Binduced apoptosis by targeting histone methyltransferase $\mathrm{EZH} 2$ depending on Myc expression. Cell Physiol Biochem. 2017;43:1188-97.

39. Qian K, Liu G, Tang Z, Hu Y, Fang Y, Chen Z, et al. The long non-coding RNA NEAT1 interacted with miR-101 modulates breast cancer growth by targeting EZH2. Arch Biochem Biophys. 2017;615:1-9.

40. Zhang L, Liao Y, Tang L. MicroRNA-34 family: a potential tumor suppressor and therapeutic candidate in cancer. J Exp Clin Cancer Res. 2019;38:53.

41. Xia W, Hou M. Mesenchymal stem cells confer resistance to doxorubicininduced cardiac senescence by inhibiting microRNA-34a. Oncol Lett. 2018;15:10037-46.

42. Di Bari M, Bevilacqua V, De Jaco A, Laneve P, Piovesana R, Trobiani L, et al. miR34a-5p mediates cross-talk between M2 muscarinic receptors and Notch-1/EGFR pathways in U87MG glioblastoma cells: implication in cell proliferation. Int J Mol Sci. 2018;19:E1631.

43. Kim JS, Kim EJ, Lee S, Tan X, Liu X, Park S, et al. miR-34a and miR-34b/c have distinct effects on the suppression of lung adenocarcinomas. Exp Mol Med. 2019;51:9.

44. Misso G, Di Martino MT, De Rosa G, Farooqi AA, Lombardi A, Campani V, et al. miR-34: a new weapon against cancer? Mol Ther Nucleic Acids. 2014; 3:e194.

45. Li CH, Xiao Z, Tong JH, To KF, Fang X, Cheng AS, et al. EZH2 coupled with HOTAIR to silence MicroRNA-34a by the induction of heterochromatin formation in human pancreatic ductal adenocarcinoma. Int J Cancer. 2017;140: $120-9$.

46. Kwon H, Song K, Han C, Zhang J, Lu L, Chen W. Epigenetic silencing of miRNA-34a in human cholangiocarcinoma via EZH2 and DNA methylation: impact on regulation of Notch pathway. Am J Pathol. 2017;187:2288-99. 